\title{
Extensive antimicrobial resistance and plasmid-carrying resistance genes in mcr-1- positive $E$. coli sampled in swine, in Guangxi, South China
}

Jingzhi Yuan, Xiaoye Wang* (D), Dali Shi, Qiang Ge, Xingxing Song, Wen Hu, Deyuan Wei, Chenling Ge, Xun Li and Chuanhuo Hu

\begin{abstract}
Background: The discovery of the superbug mcr-1-positive Escherichia coli (MCRPEC) has drew greet attention. Swine-origin multi-drug resistant MCRPEC has been a potential threat to public health and safety. However, there were few detailed studies have been reported on swine MCRPEC in Guangxi, South China.

Results: In this study, thirty-three MCRPEC strains were detected from 142 E. coli strains from 116 samples in Guangxi in 2018. Which could be classified into eight unique STs and a total of six incompatibility plasmid groups (IncFI, IncHI1, IncY, IncN, Incl1 and IncX1). After that, the susceptibility of MCRPEC isolates to 27 antimicrobial agents belonging to 17 antimicrobial categories was tested. There were nineteen $E$. coli resistant to $3 r d$ and 4 th generation cephalosporins and twelve E. coli resistant to carbapenem resistan. Importantly, the MCRPEC showed high resistance highly resistance for imipenem and meropenem, which were forbidden to use in livestock production. Three MCRPEC strains were further proved to be extensively drug-resistant (XDR), and the other isolates were multidrug-resistant (MDR). Furthermore, we found that the plasmid-carrying resistance genes coexisted with the mcr-1 gene of the MCRPEC isolates. Which were listed as follows: $\beta$-lactamase antimicrobial resistance genes e.g. ESBL genes (bla $a_{C T X-M 14}$, bla $a_{C T X-M 24}$, bla $a_{C T X-M 123}$, bla $\left.a_{O X A-1}\right)$, plasmid-mediated AmpC (pAmpC) gene (bla $\left.a_{C M Y-2}\right)$, the carbapenem resistance gene $\left(b / a_{N D M-5}\right)$, and non- $\beta$-lactamase antimicrobial resistance genes ( $q n r A$, qnrB, anrS, aac (6')-Ib-cr, tetA, tetB, sul1, sul2, floR, aadA).
\end{abstract}

Conclusion: Thirty-three mcr-1-positive E. coli isolates in Guangxi displayed a wide profile of antimicrobial resistance. Plasmid-carrying resistance genes might be the main cause of MCRPEC multidrug resistance. This study highlighted the necessity for long-term surveillance of mcr-1-positive E. coli in pigs.

Keywords: Swine-origin multi-drug resistance MCRPEC, Antimicrobial resistance, Extensively drug-resistant, Acquired antimicrobial resistance genes

\footnotetext{
*Correspondence: xywang@gxu.edu.cn

College of Animal Science and Technology, Guangxi University, Nanning

530004, P. R. China
}

(c) The Author(s). 2021 Open Access This article is licensed under a Creative Commons Attribution 4.0 International License, which permits use, sharing, adaptation, distribution and reproduction in any medium or format, as long as you give appropriate credit to the original author(s) and the source, provide a link to the Creative Commons licence, and indicate if changes were made. The images or other third party material in this article are included in the article's Creative Commons licence, unless indicated otherwise in a credit line to the material. If material is not included in the article's Creative Commons licence and your intended use is not permitted by statutory regulation or exceeds the permitted use, you will need to obtain permission directly from the copyright holder. To view a copy of this licence, visit http://creativecommons.org/licenses/by/4.0/ The Creative Commons Public Domain Dedication waiver (http://creativecommons.org/publicdomain/zero/1.0/) applies to the data made available in this article, unless otherwise stated in a credit line to the data. 


\section{Background}

Superbug infections are one of the most serious threats to public and animal health nowadays. The emergence and rapid spread of multi-drug-resistant (MDR), extensively drug-resistant (XDR) and pan-drug-resistant (PDR) bacteria has been a major public health problem worldwide [1]. MDR was defined as acquired nonsusceptibility to at least one agent in three or more antimicrobial categories, XDR was defined as nonsusceptibility to at least one agent in all but two or fewer antimicrobial categories (i.e. bacterial isolates remain susceptible to only one or two categories) and PDR was defined as non-susceptibility to all agents in all antimicrobial categories [2]. The transmissibility of antimicrobial resistance mediated by mobile plasmids was reported to be an important reason for the generation of XDR and PDR bacteria [3].

In China, colistin is the last line of defense against carbapenem-resistant Escherichia. coli (CREC) [4, 5]. However, colistin has been used in animal production in China for decades as a treatment and feed additive [6]. In 2015, the plasmid-mediated $m c r-1$ gene was first discovered in food animals in South China [7]. Subsequently, nine different $m c r$ alleles, e.g. $m c r-1$ to $m c r-9$, were found in different bacteria from many countries and regions [7-15].

Due to the extensive use of $\beta$-Lactam antimicrobial in human and veterinary medicine, the number of extended-spectrum $\beta$-lactamase (ESBL)-producing $E$. coli is increasing rapidly worldwide [16, 17]. More related studies on CREC were reported recently, for CREC is multi-drug resistant which is difficult to treat and cause a high lethality after infection [18, 19]. The occurrence of colistin resistant CREC aggravate the situation $[20,21]$.

Thus, this study aimed at investigate the phenotype of antimicrobial resistance of MCRPEC and its plasmidcarrying resistance genes from pigs in Guangxi, South China.

\section{Results}

\section{Identification of $\mathrm{mcr}-1$ positive $E$. coli (MCRPEC) isolates}

A total of $142 \mathrm{E}$. coli isolates were isolated from pigs with diarrhea/dyspnea in Guangxi in 2018. Seventy-two (50.7\%, 72/142) E. coli isolates were tested with colistin (MIC $3.5 \mathrm{mg} / \mathrm{L}$ ). To investigate the proportion of $\mathrm{mcr}$ genes in E. coli, PCR amplification was performed to test $m c r-1, m c r-2, m c r-3, m c r-4, m c r-5, m c r-6, m c r-7$, and $m c r-8.33 m c r-1$-positive $E$. coli strains detected. The percentage of MCRPEC strains accounted for 45.8\% (33/ 72 ) of colistin resistant strains and $23.2 \%$ (33/142) of all isolated strains. The thirty-three MCRPEC isolates were used for subsequent study.
The full-length $16 \mathrm{~S}$ rRNA gene sequences of the 33 MCRPEC strains were used to generate a phylogenetic tree by means of Neighbor Joining method in MEGA-X (Fig. 1). Thirty-three MCRPEC strains were classified into eight distinct STs, including ST10, ST224, ST361, ST410, ST641, ST1408, ST3345, and an unknown ST. ST10 and ST224 were the dominant STs, which accounted for $69.7 \%$ (23/33) (Fig. 1). More information about MLST was included in the supplementary materials (supplementary materials Table 2).

Multiple PCR was used to identify incompatibility plasmid groups in MCRPEC by using plasmid DNA of MCRPEC isolates. As is shown in Fig. 1, six incompatibility plasmid groups were detected, including IncFI (97.0\%, 32/33), IncHI (12.1\%, 4/33), IncY (48.5\%, 16/33), IncN $(15.2 \%, 5 / 33)$, IncI1 $(3.0 \%, 1 / 33)$ and IncX1 (6.1\%, $2 / 33)$. Ten $(30.3 \%, 10 / 33)$ MCRPEC isolates were detected to carry one incompatibility plasmid group (IncFI). Sixteen $(16 / 33,48.5 \%)$ MCRPEC isolates were detected to carry two incompatibility plasmid groups, among which eleven $(11 / 16,68.8 \%)$ isolates were the combination of IncFI and IncY, four (4/16, 25\%) isolates displayed the combination of IncHI and IncFI, and one (1/ $16,6.2 \%)$ isolate exhibited the combination of IncFI and IncX1. Six $(6 / 33,18.2 \%)$ MCRPEC isolates were detected to carry three incompatibility plasmid groups, among which four $(4 / 6,66.6 \%)$ isolates were the combination of IncFI, IncN and IncY, one $(1 / 6,16.7 \%)$ isolate was the combination of IncFI, IncI1 and IncY, and one $(1 / 16,16.7)$ isolate was the combination of IncFI, IncN and IncX1. In addition, one $(1 / 33,3.0 \%)$ MCRPEC isolate was not detected to carry incompatibility plasmid group.

\section{Antimicrobial resistance in MCRPEC}

The antimicrobial resistance proportion of thirty-three MCRPEC isolates were as follows: gentamicin $(72.7 \%$, 24/33), amikacin (48.5\%, 16/33), ceftaroline (69.7\%, 23/ $33)$, piperacillin-tazobactam $(24.2 \%, 8 / 33)$, imipenem (36.4\%, 12/33), meropenem $(24.2 \%, 8 / 33)$, cefalexin $(69.7 \%, 23 / 33)$, cefuroxime $(57.6 \%, 19 / 33)$, cefotaxime (57.6\%, 19/33), ceftriaxone $(57.6 \%, 19 / 33)$, cefepime $(39.4 \%, 13 / 33)$, cefoxitin $(0 \%, 0 / 33)$, ciprofloxacin $(75.8 \%$, 25/33), sulfadiazine $(24.2 \%, 8 / 33)$, trimethoprimsulphamethoxazole $(0 \%, 0 / 33)$, aztreonam $(24.2 \%, 8 / 33)$, ampicillin (97.0\%, 32/33), amoxicillin-clavulanic acid (0\%, 0/33), ampicillin-sulbactam (24.2\%, 8/33), chloramphenicol $(84.8 \%, 28 / 33)$, fosfomycin $(78.8 \%, 26 / 33)$, tetracycline $(100 \%, 33 / 33)$, doxycycline $(72.7 \%, 24 / 33)$, azithromycin $(57.6 \%, 19 / 33)$, polymyxin B $(100 \%, 33 / 33)$ and colistin (100\%, 33/33) (Fig. 2a). In addition, each MCRPEC isolate showed significant antimicrobial resistance (Fig. 2b). According to the results of cephalosporin susceptibility test, nineteen strains $(57.6 \%, 19 / 33)$ were resistant to the 3rd and 4th generation cephalosporins 


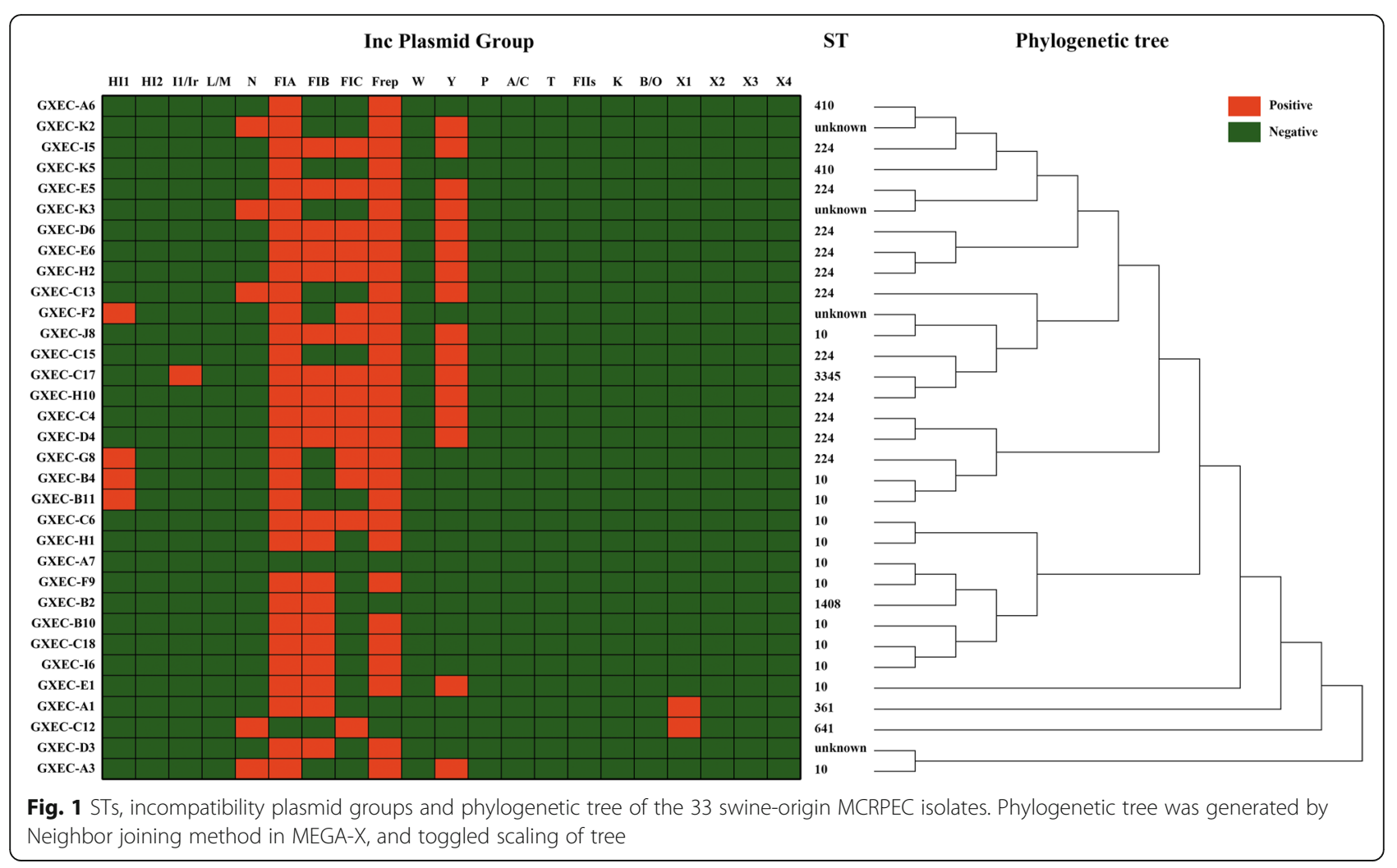

and twelve strains $(36.4 \%, 12 / 33)$ were resistant to carbapenem (Fig. 2a). According to the definition of MDR, XDR, and PDR bacteria, all 33 MCRPEC isolates were identified as MDR (Fig. 2b) [2]. Among them, three of the MDR MCRPEC isolates were identified as XDR (Fig. 2c).

\section{Coexistence of $m c r-1$ gene in plasmids with $\beta$-lactamase antimicrobial resistance genes and non- $\beta$-lactamase antimicrobial resistance genes in the MCRPEC isolates} There were 22 MCRPEC isolates harbored ESBL genes, including two $b l a_{O X A-1}$ and thirty-two $b l a_{C T X-M}$. The dominate $b l a_{C T X-M}$ gene was $b l a_{C T X-M-14}(59.4 \%, 19 / 32)$, followed by $b l a_{C T X-M-123}(37.5 \%, 12 / 32)$ and $b l a_{C T X-M-24}$ $(3.1 \%, 1 / 32)$ (Fig. 3a, b). Additionally, there were two and eight MCRPEC isolates with $b l a_{C M Y-2}$ and $b l a_{N D M-5}$ respectively. (Fig. 3a, c).

Furthermore, many non- $\beta$-lactamase antimicrobial resistance genes were also detected in MCPEC isolates, including fluoroquinolone resistance gene, tetracycline resistance genes, sulfanilamide resistance genes, aminoglycoside resistance genes and chloramphenicol resistance genes. As is shown in Fig. 3a, the rates of fluoroquinolone resistance related genes q $n r A$, qnrB, $q n r S$, and $a a c$ (6')-Ib-cr were $36.4 \%$ (12/33), 36.4\% (12/ $33), 33.3 \%(11 / 33)$, and $24.2 \%(8 / 33)$ individually. Tetracycline resistance related genes tet $A$, tet $B$, and tet $X$ accounted for $100 \%$ (33/33), $18.2 \%$ (6/33), and $0 \%(0 /$
33), respectively. Sulfanilamide resistance determinants sul1 and sul 2 accounted for $90.0 \%(30 / 33)$ and $78.8 \%$ (26/33), respectively. Aminoglycoside resistance related gene aadA (100\%, 33/33) and chloramphenicol resistance related gene floR $(100 \%, 33 / 33)$ both have $100 \%$ detection rates.

Besides, virulence genes (Enterotoxigenic E. coli and Shigatoxin-pruducing E. coli) of thirty-three MCRPEC isolates from clinical diagnostic were detected. The results were shown in Table 3 of supplementary materials. Twenty-six (26/33, 78.8\%) MCRPEC isolates were identified as pathogenic $E$. coli, of which twenty-two $(22 / 26$, 84.6\%) isolates were identified as Enteroxigenic E. coli (ETEC), three $(3 / 26,11.5 \%)$ isolates were identified as Shigatoxin-pruducing E. coli (STEC), and one (1/26, 3.9\%) isolate was both ETEC and STEC. Fifteen (15/22, 68.2\%) ETEC isolates only carried $S T b$ gene, the rest of ETEC isolates $(7 / 22,31.8 \%)$ carried $S T b$ and $L T$ genes. Three STEC isolates only carried $s t x 2 e$ gene. The ETEC/ STEC isolate carried $S T b, L T$ and $s t x 2 e$ genes.

\section{Discussion}

China became the world's lar raises and consumes about 500 million pigs a year, nearly half the world's total, and is the world's largest consumer of antibiotics [22]. The long term over antibiotics fast-growing of resistance [23]. In the challenge of antibiotic resistance, the Chinese government stipulated the veterinary drug prescription 

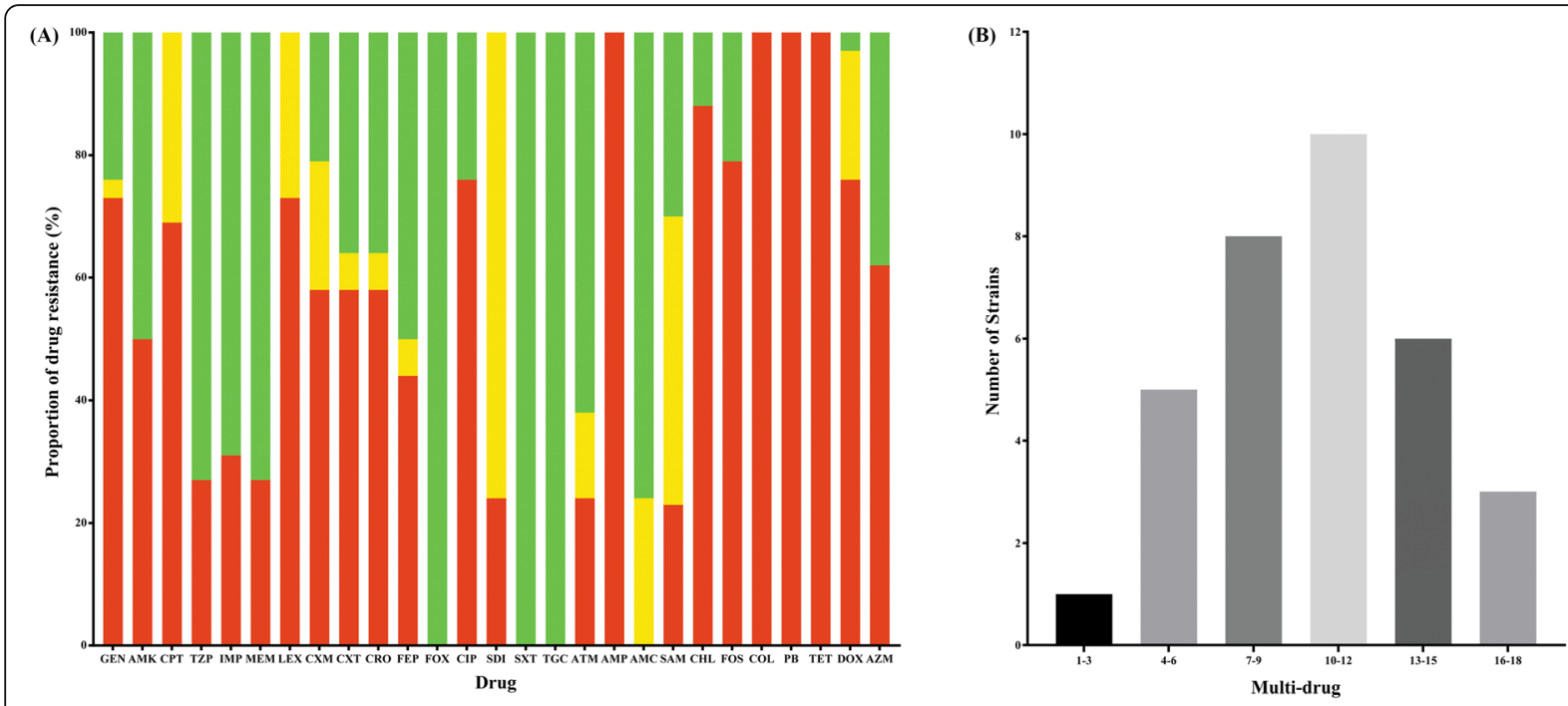

(C)
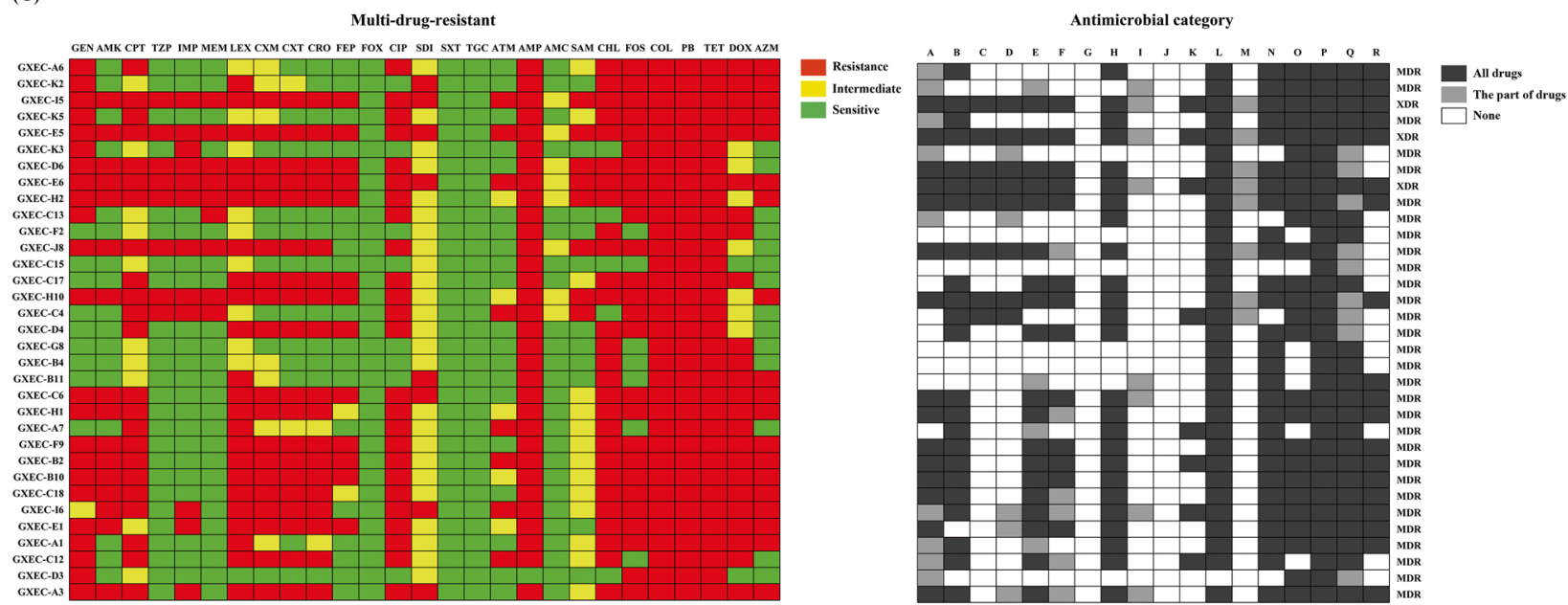

Fig. 2 Antimicrobial resistance characteristics of the 33 swine-origin MCRPEC isolates from swine E. coli. a Antimicrobial resistance proportion, the bar chart showed the percentages of the 33 MCRPEC isolates that were sensitive (green), intermediate (yellow), or resistant (red) to 27 commonly used antimicrobials. b Statistics of the multi-antimicrobial category. c The first panel consists of 27 columns representing the sensitivity (green), intermediates (yellow), or resistance (red) of 27 antimicrobial agents. The next panel contains 18 columns indicating the antimicrobial category of the 33 MCRPEC isolates, including A: aminoglycosides, B: anti-MRSA cephalosporins, C: antipseudomonal penicillins + $\beta$-lactamase inhibitors, D: carbapenems, E: 1st and 2nd generation cephalosporins, F: 3rd and 4th generation cephalosporins, G: cephamycins, H: fluoroquinolones, I: folate pathway inhibitors, J: glycylcyclines, K: monobactams, L: penicillins, M: penicillins + $\beta$-lactamase inhibitors, N: phenicols, O: phosphonic acids, P: polymyxins, Q: tetracyclines, and R: Macrolides. The colors indicate resisitance to all kinds agents of one antimicrobial category (black), resistant to some of the agents of one antimicrobial category (gray), no agent (white). The rightmost section are judgments for MDR, XDR, or PDR. Multidrug resistant (MDR) is the acquired (but not natural) insensitivity (mediated or resistant) to three or more antimicrobial agents (at least one in each category). Extensive resistant (XDR) means that it is insensitive to all antimicrobial species (at least one in each category) except for those in the 1-2 category. PDR is defined as non-susceptibility to all agents in all antimicrobial categories. The MICs of antimicrobial resistance test were putted in supplementary material Table 4

management measures in 2013 [24]. In recent years, plasmid-mediated colistin resistant genes $m c r-1$ to $m c r-9$ have been found worldwide [7-15]. Although the Chinese government began to tighten regulation of colistin in 2017, MCRPEC remains to be a chronic problem in pig farms [25-27]. Thus, we report extensive antimicrobial resistance and plasmid-carrying resistance genes in MCRP EC from pigs in Guangxi, China.
In this study, MCRPEC was found to be highly resistant to some of the $\beta$-lactam and non- $\beta$-lactam antibiotics commonly used in human medicine and veterinary medicine, such as penicillin, cephalosporins, fluoroquinolones, aminoglycosides, quinolones, sulfonamides and tetracycline. In addition, rare resistance to carbapenems in animal production such as imipenem and meropenem was also found in MCRPEC. According to veterinary 
(A) Antimicrobial resistance genes

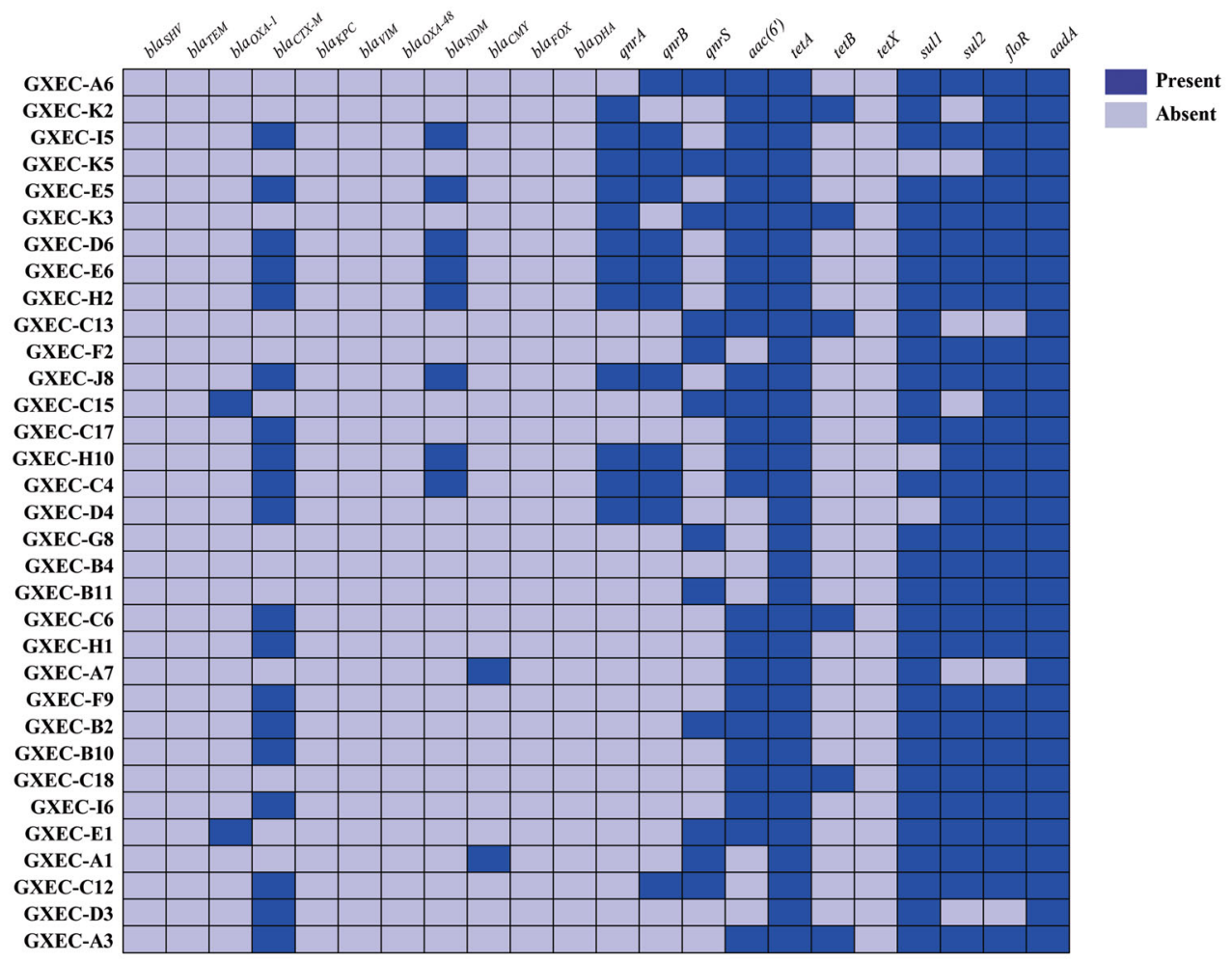

(B)

The distribution of ESBL/Anti-carbapenemase/pAmpC genes

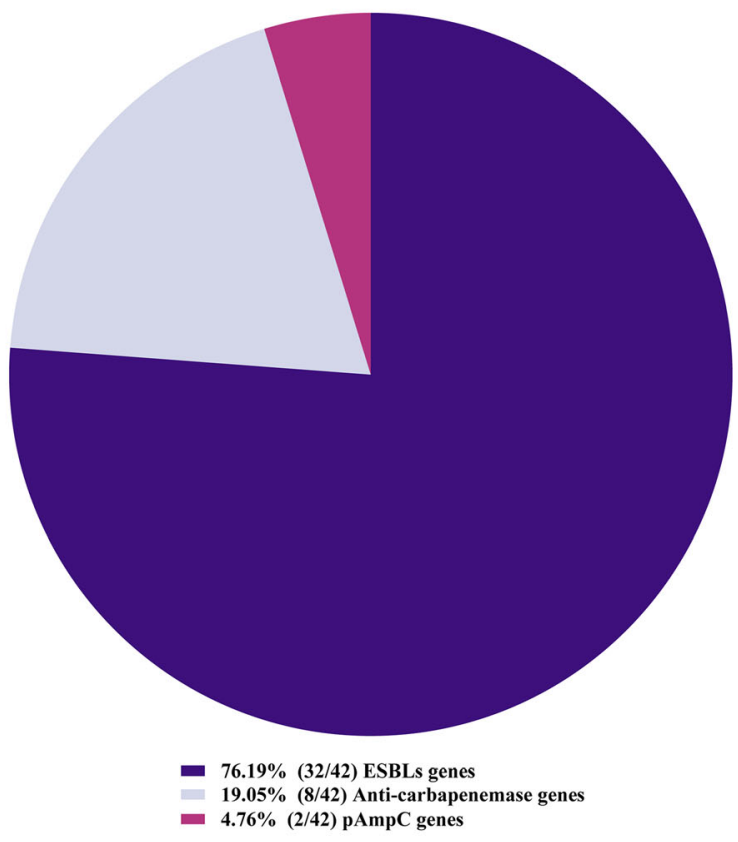

Total $=42$
(C)

The distribution of $b l a_{C T X-M}$ genes

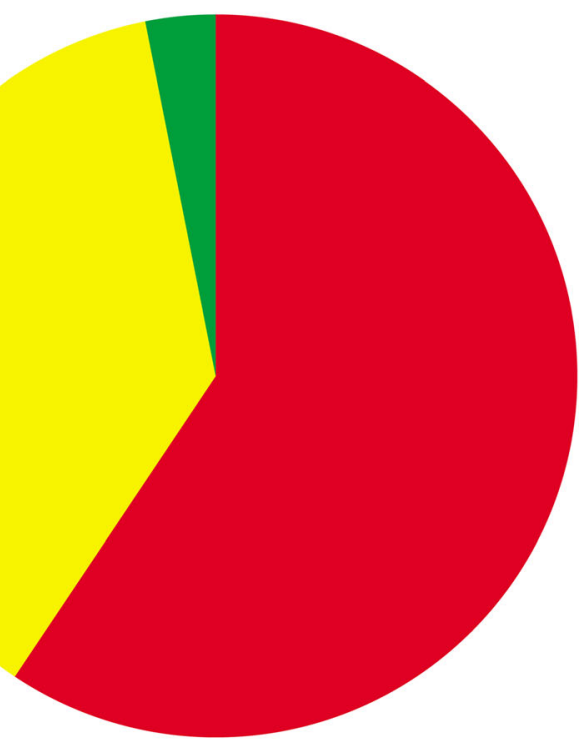

$76.20 \%(19 / 32) b^{b l a} a_{C T X-M-14}$

$19.00 \%(12 / 32)$ bla $_{C T X-M-123}$

- $3.13 \%(1 / 32) b_{C T X-M-24}$

Total $=32$

Fig. 3 Plasmid-carrying resistant genes of the 33 swine-origin MCRPEC isolates (a) The panel includes 22 columns indicating the presence or absence of plasmid-mediated resistance genes. Dark blue means presence, and light Blue means absence. $\mathbf{b}$ The distribution of the total ESBL/ Carbapenemase/pAmpC genes in the MCRPEC isolates. $\mathbf{c}$ The distribution of bla $a_{C T X-M}$ among thirty-two ESBL genes 
prescription list, imipenem and meropenem are not permitted to use in livestock production. Recently, studies have found that MCRPEC contains a "new Delhi metallo-lactam resistance gene" that is resistant to almost all $\beta$-lactam antibiotics but monobactam $[28,29]$. Thirty-three MCRPEC isolates were susceptible to tigecycline and cefoxitin. According to veterinary prescription list, tigecycline and cefoxitin are not permitted to use in livestock production [24, 25]. As the same time, Guangxi government published the notice explained tigecycline was special use level and cefoxitin was restricted use level (i.e. ordinary people is difficult to acquire) [30]. The presence of these multidrug-resistant phenotypes suggests that MCRPEC coexists with other drug-resistant genes.

Interestingly, there is evidence that ESBL E. coli has a higher level of $m c r-1$ than non-ESBL E. coli, and the rapid rise in ESBL also significantly increases the selective pressure for colistin resistance [17]. In this study, $63.6 \%$ (21/33) of MCRPEC strains were detected to contain different ESBL genes simultaneously. Among them, $\operatorname{bla}_{C T X-M}(19 / 21,90.5 \%)$ was dominant in MCRPEC plasmid mediated drug resistance genes. Among the 32 $b l a_{C T X-M}$ sequences of 19 MCRPEC isolates, $b l a_{C T X-M-14}$ gene had the highest proportion $b l a_{C T X-M}$ gene $(59.4 \%$, $19 / 32)$, followed by bla $_{\text {CTX-M-123 }}(37.5 \%, 12 / 32)$ and bla $a_{C T X-M-24}(3.1 \%, 1 / 32)$. Notably, all MCRPEC isolates carrying the $b l a_{C T X-M-14}$ genes were resistant to ciprofloxacin (Fig. 3a and c), consistent with another study [31]. Recently, several reports have shown that pig waste not only frequently carries $m c r-1$ and $b l a_{N D M}$ but also transfers these genes by affecting the environment around farms and contaminating the food chain [22, 27, 32]. Therefore, the co-existence of $m c r-1$ gene with carbapenem-resistant gene bla $_{N D M-5}(8 / 33,24.2 \%)$ in $E$. coli has drawn our attention to the spread of such superbugs in Guangxi.

E. coli is by nature sensitive to almost all clinically relevant antimicrobial agents, but this bacterium has a great capacity to accumulate resistance genes, mainly through horizontal gene transfer [33]. In this study, 11 plasmids carrying non-lactam genes were found in MCRPEC, including fluoroquinolone ( $q n r A$, qnrB, qnrS, aac (6')-Ib-cr), tetracycline $(\operatorname{tet} A, \operatorname{tet} B)$, sulfonamide (sul1, sul2), aminoglycoside (aadA), and chloramphenicol (floR), suggesting that the effect of MCRPEC on spreading non- $\beta$-lactam genes should not be underestimated.

It has previously been reported that $m c r-1$ gene was found in the conjugative plasmids, IncI2, IncFII, IncX4, IncHI1, IncHI2, IncP, IncF, and IncY [34, 35]. We also detected these incompatibility types by PCR typing. The 33 MCRPEC isolates showed six different Inc. plasmid groups including IncHI, IncIl, IncN, IncFI, IncY, IncX1. Meanwhile, we found that IncFIA and $\operatorname{IncF}_{\mathrm{rep}}$ were prevalent in 33 MCRPEC in this study. MLST results reflected that ST10 (13/33, 39.3\%) was the most common ST among the 33 MCRPEC isolates, ST224 (10/33, 30.3\%) and another ST (10/33, 30.3\%) followed (Fig. 1). Recently, a study analyzed 616 whole genomes of $m c r-1$-positive $E$. coli isolates from NCBI online database, among them ST10 was the most abundant MCRPEC strains [36].

\section{Conclusions}

The study showed that thirty-three $m c r-1$-positive $E$. coli isolates in Guangxi had a wide range of antimicrobial resistance. Plasmid-carrying resistance genes might be the main cause of MCRPEC multidrug resistance. The results indicated that many ESBL genes $\left(b l a_{C T X-M}, b l a_{O X A-1}\right)$ coexisted with mcr-1. The carbapenemase gene $b l a_{N D M-5}$ was detected in 8 MCRPEC strains. Furthermore, a number of non- $\beta$-lactam genes also coexisted with $m c r-1$ gene. Food animals and their feces are important sources of bacterial drug resistance transfer, our study highlights the necessity for long-term surveillance of $m c r-1$-positive $E$. coli in pigs.

\section{Methods}

Sample collection and detection of MCRPEC isolates

A total of 116 samples were collected from 44 pig farms that include 37 family farms and 7 swine breeding farms distributed in different towns of Guangxi, China in 2018. In which, 51 samples were collected from family farms and 65 samples were collected from swine breeding farms. These samples were collected from June 2018 to December 2018 and taken from rectal swabs or lung, intestinal tract, or lymph gland tissue collected from dead or unhealthy pigs with diarrhea or dyspnea. Before this study, these samples were sent to clinical veterinary laboratory of College of Animal Science and Technology of Guangxi University for molecular diagnosis. These farms managed about 27.8 thousand fattening pigs and 30.8 thousand breeding pigs during this study period. All fattening pigs belonged to family farms which were companies plus farmer model and small in size (number of pigs were between 400 to 1000 in one farm). Swine breeding farms adopted closed management and bigger in size (number of pigs were between 1000 to 5000 in one farm). In supplementary material Table 5 , we provided description of 116 samples origin.

First, the collected samples were inoculated with MacConkey agar for $24 \mathrm{~h}$ at $37^{\circ} \mathrm{C}$. Then all single colonies of different forms on MacConkey agar were inoculated with eosin-methylene blue agar to screen suspected $E$. coli isolates. Colonies with a purplish black color or a metallic dark green color on the eosin-methylene blue agar were considered as suspected $E$. coli and further inoculated into LB Broth (Luria-Bertani Broth) for 8-10 h at $37^{\circ} \mathrm{C}$. Colistin resistance isolates were isolated by self- 
made SuperPolymyxin medium (i.e. a mixture of $10 \mathrm{ml}$ Eosin-methylene blue agar and $35 \mu \mathrm{g}$ colistin) as previous reported [37]. The genome DNA of Colistin resistance isolates was extracted with TIANamp Bacteria DNA Kit. Colistin resistance E. coli strains were determined by $16 \mathrm{~S}$ rRNA gene sequencing and BLAST analysis (i.e. First, DNA and primers were used to amplify target fragment largely. PCR amplicons were purified by agarose gel electrophoresis and gel extraction from TIAN Gel Extraction Kit. Purified PCR products were sequenced by ABI 3730xl DNA Analyzer. SeqMan was used for sequence analysis. Finally, sequences were BLAST in NCBI.) [38]. Phylogenetic tree was generated by Neighbor joining statistical method in MEGA-X software. Thirty-three MCRPEC isolates would be tested in follow-up experiments. These isolates were from 116 pigs (un-weaned piglets, nursery piglets and sows) of 25 pig farms of different sizes distributed in 18 different towns of Guangxi, China in 2018 (Table 1). All E. coli isolates were stored in glycerol medium at $-80^{\circ} \mathrm{C}$.

The MCRPEC isolates were preliminarily screened by PCR amplification using genome DNA and special

Table 1 MCRPEC samples collection information

\begin{tabular}{|c|c|c|c|c|}
\hline Number & Town/County/City & Date (Month/Day) & Usage/Scale & Clinical symptom \\
\hline GXEC-A1 & Ning Wu, Wu Ming, Nan Ning & June 11 & Porker/800 & Diarrhea \\
\hline GXEC-A3 & Gan Xu, Wu Ming, Nan Ning & June 19 & Porker/600 & Diarrhea \\
\hline GXEC-A6 & Jin Ling, Xi Xiang Tang, Nan Ning & July 05 & Porker/570 & Diarrhea \\
\hline GXEC-A7 & Shuang Ding, Xi Xiang Tang, Nan Ning & July 14 & Porker/400 & Diarrhea \\
\hline GXEC-B2 & Shuang Ding, Xi Xiang Tang, Nan Ning & July 23 & Porker/700 & Diarrhea/Dyspnea \\
\hline GXEC-B4 & Jin Ling, Xi Xiang Tang, Nan Ning & August 02 & Porker/800 & Diarrhea/Dyspnea \\
\hline GXEC-B10 & Shuang Ding, Xi Xiang Tang, Nan Ning & August 19 & Porker/530 & Diarrhea \\
\hline GXEC-B11 & Fu cheng, Wu Ming, Nan Ning & August 27 & Porker/1019 & Diarrhea \\
\hline GXEC-C4 & Shuang Qiao, Wu Ming, Nan Ning & September 28 & Porker/750 & Diarrhea \\
\hline GXEC-C6 & Ling Tian, Ling Chuan, Gui Lin & October 15 & Porker/1000 & Diarrhea/Dyspnea \\
\hline GXEC-C12 & Shuang Qiao, Wu Ming, Nan Ning & October 15 & Porker/600 & Diarrhea \\
\hline GXEC-C13 & Sha Tian, Ping Gui, He Zhou & November 01 & Porker/900 & Diarrhea/Dyspnea \\
\hline GXEC-C15 & Shuang Ding, Xi Xiang Tang, Nan Ning & October 29 & Porker/800 & Diarrhea \\
\hline GXEC-C17 & He Jie, Ping Gui, He Zhou & November 17 & Porker/900 & Diarrhea \\
\hline GXEC-C18 & Ren Yi, Ba Bu, He Zhou & December 01 & Porker/900 & Diarrhea/Dyspnea \\
\hline GXEC-D3 & Sha Tian, Ping Gui, He Zhou & November 18 & Porker/700 & Diarrhea \\
\hline GXEC-D4 & Kui Yang, Xing Ye, Yu Lin & December 16 & Porker/830 & Diarrhea \\
\hline GXEC-D6 & Long Meng, Pu Bei, Qin Zhou & December 20 & Porker/750 & Diarrhea/Dyspnea \\
\hline GXEC-E1 & Shuang Qiao, Wu Ming, Nan Ning & October 11 & Un-weaned piglet/700 & Diarrhea \\
\hline GXEC-E5 & Shuang Qiao, Wu Ming, Nan Ning & October 11 & Un-weaned piglet/700 & Diarrhea \\
\hline GXEC-E6 & Shuang Qiao, Wu Ming, Nan Ning & October 11 & Sow/700 & Diarrhea \\
\hline GXEC-F2 & Da Hua, He Chi & November 03 & Un-weaned piglet/5000 & Diarrhea \\
\hline GXEC-F9 & Da Hua, He Chi & November 03 & Un-weaned piglet/5000 & Diarrhea \\
\hline GXEC-G8 & Fu Cheng, Wu Ming, Nan Ning & November 11 & Un-weaned piglet/1500 & Diarrhea \\
\hline GXEC-H1 & Lu Zhai, Liu Zhou & November 22 & Un-weaned piglet/1500 & Diarrhea \\
\hline GXEC-H2 & Lu Zhai, Liu Zhou & November 22 & Un-weaned piglet/1500 & Diarrhea \\
\hline GXEC-H10 & Lu Zhai, Liu Zhou & November 22 & Sow/1500 & Diarrhea \\
\hline GXEC-15 & Feng Huang, Xing Bin, Lai bin & December 06 & Sow/5000 & Diarrhea \\
\hline GXEC-16 & Feng Huang, Xing Bin, Lai bin & December 06 & Sow/5000 & Diarrhea \\
\hline GXEC-J8 & Xing An, Xing An, Gui Lin & December 15 & Un-weaned piglet/1500 & Diarrhea \\
\hline GXEC-K2 & San Jie, Ling Chuan, Gui Lin & December 22 & Un-weaned piglet/1000 & Diarrhea \\
\hline GXEC-K3 & San Jie, Ling Chuan, Gui Lin & December 22 & Un-weaned piglet/1000 & Diarrhea \\
\hline GXEC-K5 & San Jie, Ling Chuan, Gui Lin & December 22 & Sow/1000 & Diarrhea \\
\hline
\end{tabular}

Un-weaned piglets were concentrated on less than 20 days. Nursery piglets were concentrated on 40 to 60 days. Sows were less than 100 days old 
primer pairs for the $m c r-1, m c r-2, m c r-3, m c r-4, m c r-$ 5, $m c r-6, m c r-7$, and $m c r-8$ genes (supplementary material Tables 1), [39, 40]. To identify MCRPEC strains, the $m c r-1$ gene sequences in the $E$. coli strains were determined by direct sequencing from the PCR products and BLAST analysis [38].

\section{Detection of multilocus sequence typing (MLST) and incompatibility plasmid groups}

MLST analysis was performed by PCR amplicons of seven housekeeping genes, namely $a d k$, fum $C$, gyrB, $i c d, m d h$, purA, and recA using genome DNA. PCR amplicons were sequenced after purified by agarose gel electrophoresis and gel extraction by TIAN Gel Extraction Kit. The gene sequences for seven housekeeping genes were uploaded to the EnteroBase database to obtain the sequence type (ST) of corresponding E. coli isolate [41].

Incompatibility plasmid groups were assigned by PCRbased replicon types (HI1, HI2, I1, L/M, N, FIA, FIB, FIC, Frep, W Y, P, A/C, T, FIIS, K/B, B/O) [42]. Additional PCRs were performed for the IncX (X1, X2, X3,
X4) replicon types [43]. The primers of house genes and replicon, and PCR reaction conditions were included in supplementary material Table 1.

\section{Antimicrobial susceptibility testing}

According to the European Society of Clinical Microbiology and Infectious Diseases (ESCMID) consensus, a total of 27 commonly used human antimicrobials from 18 antimicrobial categories were selected in this study, including gentamicin, amikacin, ceftaroline, piperacillin-tazobactam, imipenem, meropenem, cefalexin, cefuroxime, cefotaxime, ceftriaxone, cefepime, cefoxitin, ciprofloxacin, sulfadiazine, trimethoprimsulphamethoxazole, aztreonam, ampicillin, amoxicillinclavulanic acid, ampicillin-sulbactam, chloramphenicol, fosfomycin, tetracycline, doxycycline, azithromycin, polymyxin B and colistin (Table 2), [2]. Minimum inhibitory concentrations (MICs) were determined by using the agar microdilution (Mueller-Hinton Agar) method according to the Clinical and Laboratory Standards Institute [44]. The MICs of each drug were measured and recorded. E. coli ATCC25922 was used as a quality control. Resistant breakpoints of other

Table 2 The antimicrobial agents for 17 antimicrobial categories used to define the E. coli antimicrobial resistance

\begin{tabular}{|c|c|}
\hline Antimicrobial category & Antimicrobial agent \\
\hline Aminoglycosides & $\begin{array}{l}\text { Gentamicin (GEN) } \\
\text { Amikacin (AMK) }\end{array}$ \\
\hline Anti-MRSA cephalosporin & Ceftaroline (CPT) \\
\hline Antipseudomonal penicillin $+\beta$-lactamase inhibitor & Piperacillin/tazobactam (TZP) \\
\hline Carbapenem & $\begin{array}{l}\text { Imipenem (IMP) } \\
\text { Meropenem (MEM) }\end{array}$ \\
\hline Non-extended spectrum cephalosporins & $\begin{array}{l}\text { Cefalexin (LEX) } \\
\text { Cefuroxime (CXM) }\end{array}$ \\
\hline 3rd and 4th generation cephalosporins & $\begin{array}{l}\text { Cefotaxime (CTX) } \\
\text { Ceftriaxone (CRO) } \\
\text { Cefepime (FEP) }\end{array}$ \\
\hline Cephamycin & Cefoxitin (FOX) \\
\hline Fluoroquinolone & Ciprofloxacin (CIP) \\
\hline Folate pathway inhibitor & $\begin{array}{l}\text { Sulfadiazine (SDI) } \\
\text { Trimethoprim/sulfamethoxazole (SXT) }\end{array}$ \\
\hline Glycylcycline & Tigecycline (TGC) \\
\hline Monobactam & Aztreonam (ATM) \\
\hline Penicillin & Ampicillin (AMP) \\
\hline Penicillin+ $\beta$-lactamase inhibitors & $\begin{array}{l}\text { Amoxicillin/clavulanicacid (AMC) } \\
\text { Ampicillin-sulbactam (SAM) }\end{array}$ \\
\hline Phenicol & Chloramphenicol (CHL) \\
\hline Phosphonic acid & Fosfomycin (FOS) \\
\hline Polymyxins & $\begin{array}{l}\text { PolymyxinB (PB) } \\
\text { Colistin (COL) }\end{array}$ \\
\hline Tetracycline & $\begin{array}{l}\text { Tetracycline (TET) } \\
\text { Doxycycline (DOX) }\end{array}$ \\
\hline Macrolides & Azithromycin (AZM) \\
\hline
\end{tabular}


antimicrobial abided by the CLSI-M100 document [45]. CLSI breakpoints are not available for colistin and cefalexin. So, in this study, we adopted the European Committee on Antimicrobial Susceptibility Testing Resistant/Susceptible breakpoints for determine colistin and cefalexin MICs. MICs of $\leq 2 \mathrm{mg} / \mathrm{L}$ and $\leq$ $16 \mathrm{mg} / \mathrm{L}$ are considered as susceptible (S) for colistin and cephalexin, respectively, according to the EUCAST guidelines [46].

\section{Molecular identification of ESBL, $\mathrm{pAmpC}$, and carbapenem resistance genes}

The ESBL, plasmid-mediated AmpC (pAmpC), and carbapenem genes were detected by multiplex PCR in plasmid DNA of MCRPEC isolates. The ESBL genes $\left(b l a_{C T X-M}, \quad b l a_{T E M}, b l a_{O X A-1}\right.$, and $\left.b l a_{S H V}\right)$, plasmidmediated AmpC (pAmpC) genes (bla ${ }_{C M Y}, b l a_{F O X}$, $\left.b l a_{D H A}\right)$, and carbapenem resistance genes $\left(b l a_{N D M}\right.$, $b l a_{K P C}, b l a_{O X A-48}$, and $\left.b l a_{I M P}\right)$ were amplified using specific primers, as previously reported [47]. The DNA sequences for the ESBL, plasmid-mediated AmpC (pAmpC), and carbapenemase genes were determined by using BLAST analysis [38]. The primers of $\beta$-lactamase resistance genes and PCR reaction conditions were included in supplementary material Table 1.

\section{Detection of non- $\beta$-lactamase antimicrobial resistance genes}

The non- $\beta$-lactamase antimicrobial resistance genes were detected by PCR in plasmid DNA of MCRPEC isolates. The special primers included plasmid-encoded fluoroquinolone resistance genes ( $q n r A$, qnrB, qnrS, aac (6')-Ibcr) [48], tetracycline resistance genes (tet $A$, tet $B$, tet $X)$ [49], sulfonamide resistance genes (sul1, sul2), aminoglycoside resistance genes $(\operatorname{aad} A)$, and chloramphenicol resistance genes $($ floR $)$, respectively. The primers of non- $\beta$ lactamase resistance genes and PCR reaction conditions were included in supplementary material Table 1.

\section{Supplementary Information}

The online version contains supplementary material available at https://doi. org/10.1186/s12917-021-02758-4.

\section{Additional file 1.}

\section{Abbreviations}

MCRPEC: Mcr-1-positive Escherichia coli; MDR: Multi-drug-resistance; XDR: Extensively-drug-resistant; PDR: Pan-drug-resistant; CREC: Carbapenem resistant Escherichia coli; ESBL: Extended-spectrum $\beta$-lactam; pAmpC: Plasmidmediated AmpC

\section{Acknowledgements}

Not applicable.

\section{Authors' contributions}

JY carried out the experiment, analyzed samples and statistical data and wrote the manuscript. DS, QG and XS participated in experiment. WH, DW and $\mathrm{CG}$ isolated samples. $\mathrm{XL}$ and $\mathrm{CH}$ contributed to revise the manuscript. XW was corresponding authors, who mainly designed the study and supervised the whole program. All authors have read and approved the final manuscript.

\section{Funding}

This research was supported by grant from the National Natural Science Foundation of China (31502079 and 31660700), National Natural Science Foundation of Guangxi (2017GXNSFAA198071) and Science and Technology Development Program of Nanning (20180526). The Purchase of experimental consumables in this study were supported by Grant 31502079.The sample collections and gene sequence were supported by Grant 2017GXNSFAA198071.The data analysis were aided by Grant 31660700.And the writing of this manuscript was supported by Grant 20180526.

\section{Availability of data and materials}

The datasets generated and/or analysed during the current study are available in the [Github] repository, [https://github.com/YuanJZ1994/rawdata.git].

All data generated or analysed during this study are included in this published article [and its supplementary information files].

\section{Ethics approval and consent to participate}

This study was approved by Animal Experimental Ethical Committee of Guangxi University. This study was approved by College of Animal Science and Technology of Guangxi University and all relevant companies. This study obtained consent from all farms' owners. This study conformed to the legal requirements of Guangxi Zhuang Autonomous Region. The serial number of Animal Experimental Ethical Inspection Form was GXU2018-053.

\section{Consent for publication}

Not applicable.

\section{Competing interests}

The authors declare that they have no conflict of interest.

Received: 23 July 2019 Accepted: 12 January 2021

Published online: 18 February 2021

\section{References}

1. Wang $Q$, Wang $X$, Wang J, Ouyang $P$, Jin C, Wang R, Zhang Y, Jin L, Chen H, Wang Z, et al. Phenotypic and Genotypic Characterization of Carbapenemresistant Enterobacteriaceae: Data From a Longitudinal Large-scale CRE Study in China (2012-2016). Clin Infect Dis. 2018;67(suppl_2):S196-205.

2. Huang YH, Chou SH, Liang SW, Ni CE, Lin YT, Huang YW, Yang TC. Emergence of an XDR and carbapenemase-producing hypervirulent Klebsiella pneumoniae strain in Taiwan. J Antimicrob Chemother. 2018;73(8): 2039-46.

3. Magiorakos AP, Srinivasan A, Carey RB, Carmeli Y, Falagas ME, Giske CG, Harbarth S, Hindler JF, Kahlmeter G, Olsson-Liljequist B, et al. Multidrugresistant, extensively drug-resistant and pandrug-resistant bacteria: an international expert proposal for interim standard definitions for acquired resistance. Clin Microbiol Infect. 2012;18(3):268-81.

4. Dolejska M, Papagiannitsis CC. Plasmid-mediated resistance is going wild. Plasmid. 2018;99:99-111.

5. Shen Z, Hu Y, Sun Q, Hu F, Zhou H, Shu L, Ma T, Shen Y, Wang Y, Li J, et al. Emerging carriage of NDM-5 and MCR-1 in Escherichia coli from healthy people in multiple regions in China: a cross sectional observational Study. EClinicalMedicine. 2018;6:11-20.

6. Collignon P, Voss A. China, what antibiotics and what volumes are used in food production animals? Antimicrob Resist Infect Control. 2015;4:16.

7. Liu Y-Y, Wang Y, Walsh TR, Yi L-X, Zhang R, Spencer J, Doi Y, Tian G, Dong $B$, Huang $X$, et al. Emergence of plasmid-mediated colistin resistance mechanism MCR-1 in animals and human beings in China: a microbiological and molecular biological study. Lancet Infect Dis. 2016;16(2): $161-8$.

8. Xavier BB, Lammens C, Ruhal R, Kumar-Singh S, Butaye P, Goossens H, Malhotra-Kumar S. Identification of a novel plasmid-mediated colistinresistance gene, mcr-2, in Escherichia coli, Belgium, June 2016. Euro Surveill. 2016;21(27):30280-6. 
9. Roer L, Hansen F, Stegger M, Sönksen UW, Hasman H, Hammerum AM. Novel mcr-3 variant, encoding mobile colistin resistance, in an ST131 Escherichia coli isolate from bloodstream infection, Denmark, 2014. Euro Surveill. 2017;22(31):30584-5.

10. Borowiak M, Fischer J, Hammerl JA, Hendriksen RS, Szabo I, Malorny B. Identification of a novel transposon-associated phosphoethanolamine transferase gene, mcr-5, conferring colistin resistance in d-tartrate fermenting Salmonella enterica subsp. enterica serovar Paratyphi B. J Antimicrob Chemother. 2017;72(12):3317-24.

11. Yang YQ, Li YX, Lei CW, Zhang AY, Wang HN. Novel plasmid-mediated colistin resistance gene mcr-7.1 in Klebsiella pneumoniae. J Antimicrob Chemother. 2018;73(7):1791-5.

12. AbuOun M, Stubberfield EJ, Duggett NA, Kirchner M, Dormer L, NunezGarcia J, Randall LP, Lemma F, Crook DW, Teale C, et al. mcr-1 and mcr-2 variant genes identified in Moraxella species isolated from pigs in Great Britain from 2014 to 2015. J Antimicrob Chemother. 2017;72(10):2745-9.

13. Wang X, Wang Y, Zhou Y, Li J, Yin W, Wang S, Zhang S, Shen J, Shen Z, Wang Y. Emergence of a novel mobile colistin resistance gene, mcr-8, in NDMproducing Klebsiella pneumoniae. Emerg Microbes Infect. 2018;7(1):122

14. Yin W, Li H, Shen Y, Liu Z, Wang S, Shen Z, Zhang R, Walsh TR, Shen J, Wang Y. Novel Plasmid-Mediated Colistin Resistance Gene mcr-3 in Escherichia coli. mBio. 2017;8(3):e00543-17.

15. Carroll LM, Gaballa A, Guldimann C, Sullivan G, Henderson LO, Wiedmann M. Identification of Novel Mobilized Colistin Resistance Gene mcr-9 in a Multidrug-Resistant, Colistin-Susceptible Salmonella enterica Serotype Typhimurium Isolate. mBio. 2019;10(3):e00853-19.

16. Dandachi I, Chabou S, Daoud Z, Rolain JM. Prevalence and emergence of extended-Spectrum cephalosporin-, Carbapenem- and Colistin-Resistant Gram Negative Bacteria of Animal Origin in the Mediterranean Basin. Front Microbiol. 2018;9:2299.

17. Wu C, Wang Y, Shi X, Wang S, Ren H, Shen Z, Wang Y, Lin J, Wang S. Rapid rise of the ESBL and mcr-1 genes in Escherichia coli of chicken origin in China, 2008-2014. Emerg Microbes Infect. 2018;7(1):30.

18. Alotaibi FE, Bukhari EE, Al-Mohizea MM, Hafiz T, Essa EB, AlTokhais YI. Emergence of carbapenem-resistant Enterobacteriaceae isolated from patients in a university hospital in Saudi Arabia. Epidemiology, clinical profiles and outcomes. J Infect Public Health. 2017;10(5):667-73.

19. Zhang R, Liu L, Zhou H, Chan EW, Li J, Fang Y, Li Y, Liao K, Chen S. Nationwide surveillance of clinical Carbapenem-resistant Enterobacteriaceae (CRE) strains in China. EBioMedicine. 2017;19:98-106.

20. van Duin D, Lok JJ, Earley M, Cober E, Richter SS, Perez F, Salata RA, Kalayjian RC, Watkins RR, Doi Y, et al. Colistin versus Ceftazidime-avibactam in the treatment of infections due to Carbapenem-resistant Enterobacteriaceae. Clin Infect Dis. 2018;66(2):163-71.

21. Mediavilla JR, Patrawalla A, Chen L, Chavda KD, Mathema B, Vinnard C, Dever LL, Kreiswirth BN. Colistin- and Carbapenem-Resistant Escherichia coli Harboring mcr-1 and blaNDM-5, Causing a Complicated Urinary Tract Infection in a Patient from the United States. mBio. 2016;7(4):e01191-16.

22. Pharmaceuticals LC. China's lakes of pig manure spawn antibiotic resistance. Science. 2015;347(6223):704.

23. Hvistendahl M. Public health. China takes aim at rampant antibiotic resistance. Science. 2012;336(6083):795.

24. Announcement No. 1997 [2013] Of the ministry of agriculture of China. http:// www.moa.gov.cn/gk/tzgg_1/gg/201612/t20161201_5386856.htm. 1 Dac 2019.

25. Announcement No. 2471 [2016] of the Ministry of agriculture of China. http://www.moa.gov.cn/gk/tzgg_1/gg/201612/t20161201_5386856.htm. 1 Dac 2019.

26. Tong H, Liu J, Yao X, Jia H, Wei J, Shao D, Liu K, Qiu Y, Ma Z, Li B. High carriage rate of $\mathrm{mcr}-1$ and antimicrobial resistance profiles of mcr-1-positive Escherichia coli isolates in swine faecal samples collected from eighteen provinces in China. Vet Microbiol. 2018;225:53-7.

27. Xia X, Wang Z, Fu Y, Du XD, Gao B, Zhou Y, He J, Wang Y, Shen J, Jiang H, et al. Association of colistin residues and manure treatment with the abundance of mcr-1 gene in swine feedlots. Environ Int. 2019;127:361-70.

28. Yong D, Toleman MA, Giske CG, Cho HS, Sundman K, Lee K, Walsh TR. Characterization of a new metallo-beta-lactamase gene, Bla (NDM-1), and a novel erythromycin esterase gene carried on a unique genetic structure in Klebsiella pneumoniae sequence type 14 from India. Antimicrob Agents Chemother. 2009;53(12):5046-54.

29. Kong LH, Lei CW, Ma SZ, Jiang W, Liu BH, Wang YX, Guan R, Men S, Yuan QW, Cheng GY, et al. Various Sequence Types of Escherichia coli Isolates
Coharboring blaNDM-5 and mcr-1 Genes from a Commercial Swine Farm in China. Antimicrob Agents Chemother. 2017;61(3):e02167-16.

30. Announcement No. 80 [2012] of the Health Commission of Guangxi Zhuang Autinomous Region. http://wsjkw.gxzf.gov.cn/jgxx/cszc/yzc/ zhengcefagui/2012/0801/1995.html. 13 May 2020.

31. Patil S, Chen X, Lian M, Wen F. Phenotypic and genotypic characterization of multi-drug-resistant Escherichia coli isolates harboring blaCTX-M group extended-spectrum beta-lactamases recovered from pediatric patients in Shenzhen, southern China. Infect Drug Resist. 2019;12:1325-32.

32. Atterby C, Osbjer K, Tepper V, Rajala E, Hernandez J, Seng S, Holl D, Bonnedahl J, Borjesson S, Magnusson U, et al. Carriage of carbapenemaseand extended-spectrum cephalosporinase-producing Escherichia coli and Klebsiella pneumoniae in humans and livestock in rural Cambodia; gender and age differences and detection of blaOXA-48 in humans. Zoonoses Public Health. 2019:66(6):603-17.

33. Poirel L, Madec JY, Lupo A, Schink AK, Kieffer N, Nordmann P, Schwarz S. Antimicrobial Resistance in Escherichia coli. Microbiol Spectr. 2018;6(4).

34. Wang Q, Sun J, Li J, Ding Y, Li XP, Lin J, Hassan B, Feng Y. Expanding landscapes of the diversified mcr-1-bearing plasmid reservoirs. Microbiome. 2017;5(1):70.

35. Zhang C, Feng Y, Liu F, Jiang H, Qu Z, Lei M, Wang J, Zhang B, Hu Y, Ding J, et al. A Phage-Like IncY Plasmid Carrying the mcr-1 Gene in Escherichia coli from a Pig Farm in China. Antimicrob Agents Chemother. 2017;61(3): e02035-16.

36. Zhuge $X$, Ji Y, Tang F, Sun Y, Jiang M, Hu W, Wu Y, Xue F, Ren J, Zhu W, et al. Population structure and antimicrobial resistance traits of avian-origin mcr-1-positive Escherichia coli in eastern China, 2015 to 2017. Transbound Emerg Dis. 2019;66(5):1920-9.

37. Nordmann P, Jayol A, Poirel L. A universal culture medium for screening Polymyxin-resistant gram-negative isolates. J Clin Microbiol. 2016;54(5): 1395-9.

38. National Center of Biotechnology Information. http://www.ncbi.nlm.nih.gov. 15 May 2019

39. Rebelo AR, Bortolaia V, Kjeldgaard JS, Pedersen SK, Leekitcharoenphon P, Hansen IM, Guerra B, Malorny B, Borowiak M, Hammerl JA, et al. Multiplex PCR for detection of plasmid-mediated colistin resistance determinants, mcr-1, mcr-2, mcr-3, mcr-4 and mcr-5 for surveillance purposes. Euro Surveill. 2018;23(6):17-00672.

40. Yang F, Shen C, Zheng X, Liu Y, El-Sayed Ahmed MAE, Zhao Z, Liao K, Shi Y, Guo $X$, Zhong R, et al. Plasmid-mediated colistin resistance gene mcr-1 in Escherichia coli and Klebsiella pneumoniae isolated from market retail fruits in Guangzhou, China. Infect Drug Resist. 2019;12:385-9.

41. Zhou Z, Alikhan NF, Mohamed K, Fan Y, Agama Study G, Achtman M. The EnteroBase user's guide, with case studies on Salmonella transmissions, Yersinia pestis phylogeny, and Escherichia core genomic diversity. Genome Res. 2020;30(1):138-52

42. Carattoli A, Bertini A, Villa L, Falbo V, Hopkins KL, Threlfall EJ. Identification of plasmids by PCR-based replicon typing. J Microbiol Methods. 2005;63(3): 219-28

43. Johnson TJ, Bielak EM, Fortini D, Hansen LH, Hasman H, Debroy C, Nolan LK, Carattoli A. Expansion of the IncX plasmid family for improved identification and typing of novel plasmids in drug-resistant Enterobacteriaceae. Plasmid. 2012;68(1):43-50.

44. CLSI. Performance standards for antimicrobial susceptibility testing. Wayne, PA: CLSI Supplement M100 Clinical and Laboratory Standards Institute; 2018.

45. CLSI-M100. http://www.clsi.org/m100/. 20 Apr 2019.

46. European Committee on Antimicrobial Susceptibility Testing. http://www. eucast.org/clinical_breakpoints/. 20 Apr 2019.

47. Poirel L, Walsh TR, Cuvillier V, Nordmann P. Multiplex PCR for detection of acquired carbapenemase genes. Diagn Microbiol Infect Dis. 2011;70(1):11923.

48. Cattoir V, Poirel L, Rotimi V, Soussy CJ, Nordmann P. Multiplex PCR for detection of plasmid-mediated quinolone resistance qnr genes in ESBLproducing enterobacterial isolates. J Antimicrob Chemother. 2007;60(2):394-7.

49. He T, Wang R, Liu D, Walsh TR, Zhang R, Lv Y, Ke Y, Ji Q, Wei R, Liu Z, et al. Emergence of plasmid-mediated high-level tigecycline resistance genes in animals and humans. Nat Microbiol. 2019;4(9):1450-6.

\section{Publisher's Note}

Springer Nature remains neutral with regard to jurisdictional claims in published maps and institutional affiliations. 